\title{
Isolierung von MRSA-Patienten: Wie lange noch?
}

\author{
Roland Schulze-Röbbecke
}

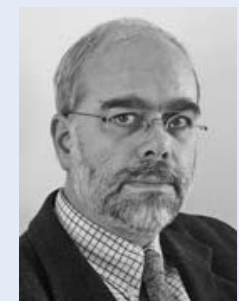

Roland SchulzeRöbbecke

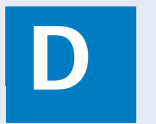

ie Argumente für die räumliche Isolierung stationärer Patienten mit Methicillinresistentem Staphylococcus aureus (MRSA) verlieren zunehmend an Überzeugungskraft. In den 1990er-Jahren stellten MRSA-Infektionen eine Herausforderung dar, weil zu ihrer Therapie oft nur noch Vancomycin und Teicoplanin zur Verfügung standen. Mit der Zulassung neuer Antibiotika hat sich diese Situation seit der Jahrtausendwende jedoch deutlich entschärft.

Das bei Ausbrüchen erprobte Konzept des aktiven Erreger-Screenings und der Isolierung positiv getesteter Patienten (Screening- und Isolierungs-Konzept) wurde seit den 1990er-Jahren in Deutschland [1] und anderswo als Maßnahme auch gegen die endemische Verbreitung von MRSA empfohlen. Einige Studien schienen den Erfolg des Screening- und IsolierungsKonzepts zu belegen. Hierbei handelte es sich jedoch durchweg um Studien von niedriger Beweiskraft, insbesondere um monozentrische, nicht randomisierte, „Vorher-nachher-“ Beobachtungsstudien. Eine multizentrische Interventionsstudie [2] schien dann 2011 einen deutlichen Beleg für die Effektivität des Screening- und Isolierungs-Konzepts zu liefern; eine unabhängige Datenauswertung zeigte anschließend jedoch, dass nur ein sehr kleiner Anteil des in dieser Studie berichteten Erfolgs diesem Konzept zuzuschreiben war [3].

Kürzlich publizierten Huang et al. die Ergebnisse einer multizentrischen, Cluster-randomisierten Studie [4], in der die Effektivität des Screening- und IsolierungsKonzepts mit und ohne Dekolonisationsmaßnahmen bei erwachsenen Intensivpatienten von 43 Krankenhäusern überprüft wurde. Das Konzept erwies sich jedoch auch hier als ineffektiv. Gezielte Dekolonisationsmaßnahmen nur bei MRSA-positiven Patienten, insbesondere aber allgemeine Dekolonisationsmaßnahmen bei allen Patienten (ohne zusätzliche Screening- und Isolierungsmaßnahmen) führten dagegen zu einer deutlichen Verminderung klinischer MRSANachweise und des Nachweises anderer Erreger in Blutkulturen.
Kommentiert wird diese Studie in einem Editorial von Michael B. Edmond und Richard P. Wenzel [5]. Bemerkenswert ist dieses Editorial nicht nur, weil das Screening- und Isolierungs-Konzept darin als „abgeschlossener Fall“ bezeichnet wird sondern auch, weil es sich bei Wenzel um den Doyen der US-amerikanischen Krankenhaushygiene handelt.

Edmond und Wenzel unterscheiden 2 grundlegend verschiedene Präventionsstrategien, die „vertikale“ und die "horizontale“:

- Als typisches Beispiel für die vertikale Präventionsstrategie wird das Screening- und Isolierungs-Konzept bezeichnet. Die Strategie dient der Verminderung von Infektions- und Kolonisationsfällen durch spezifische Erreger, beinhaltet oft ein mikrobiologisches Erreger-Screening und zeichnet sich durch hohe Kosten und hohen Ressourcen-Verbrauch aus [6]. Vertikalen Konzepten liegt das bei Ausbrüchen bewährte Prinzip der Ausschließlichkeit zugrunde: Ein bestimmter Erreger tritt vor allen anderen Erregern in den Vordergrund und wird mit besonderen Maßnahmen bekämpft.

- Horizontale Präventionsstrategien werden dagegen auf die gesamte zu schützende Population angewendet und richten sich gegen alle Erreger, die mit einem bestimmten Mechanismus übertragen werden. Die horizontale Vorgehensweise entspricht mehr den Interessen des Patienten, der nicht nur vor einer Infektion (z. B. durch MRSA) sondern vor allen Infektionen geschützt sein möchte. Zu den horizontalen Präventionsmaßnahmen zählen z.B. die Händehygiene, alle anderen Elemente der Standard- oder Basishygiene [7] sowie Interventionsbündel [8]. Obwohl Edmond und Wenzel das Konzept allgemeiner Dekolonisationsmaßnahmen wegen der damit einhergehenden Resistenzrisiken kritisch betrachten, interpretieren sie die Ergebnisse der Studie von Huang et al. [4] als Beleg für den Erfolg des horizontalen Präventionsprinzips.

Angesichts des ungenügenden Effektivitätsnachweises fordern Edmond und Wenzel in ihrem Editorial die Aufgabe des Screening- und Isolierungs-Konzepts zur Bekämpfung der endemischen Verbreitung von MRSA und verweisen dabei auf die negativen Folgen dieser 
Maßnahme für die betroffenen Patienten [9]. Sie kritisieren die Übertragung des Konzepts auch auf andere multiresistente Erreger und betonen die Bedeutung horizontaler Interventionsmaßnahmen, um nicht nur gegen die Problemkeime von heute, sondern auch gegen die von morgen vorzugehen.

Die Diskussion um „vertikale“ und „horizontale“ Maßnahmen zur Verhinderung der MRSA-Verbreitung ist in Deutschland nicht neu, wenn auch mit anderer Begrifflichkeit. So publizierten Kappstein et al. vor 4 Jahren eine monozentrische, beobachtende Verlaufsstudie [10], aus deren Ergebnissen sie schlussfolgern, dass zur Prävention der MRSA-Verbreitung im Krankenhaus die („horizontale“) Standardhygiene dem („vertikalen“) Screening- und Isolierungs-Konzept mindestens ebenbürtig ist. Eine unvoreingenommene wissenschaftliche Debatte war damals nicht möglich, ist heute aber angesichts der teuren und vielerorts an ihre Grenzen stoßenden Isolierungsstrategie nötiger denn je.

Im Rahmen der Ausbruchsbekämpfung spielt das Screening- und Isolierungs-Konzept nach wie vor eine zentrale Rolle. Für die meist vorherrschenden endemischen Verhältnisse spricht die aktuelle Studienlage inzwischen jedoch gegen einen nennenswerten präventiven Effekt dieses Konzepts. In einer aktuellen evidenzbasierten Leitlinie könnte es daher bestenfalls noch mit der Kategorie III versehen werden („keine Empfehlung, ungeklärte Frage“).

\section{Literatur}

1 Kommission für Krankenhaushygiene und Infektionsprävention am Robert Koch-Institut. Empfehlung zur Prävention und Kontrolle von Methicillin-resistenten Staphylococcus aureusStämmen (MRSA) in Krankenhäusern und anderen medizinischen Einrichtungen. Bundesgesundheitsbl 1999; 42: 954 958

2 Jain R, Kralovic SM, Evans ME et al. Veterans Affairs initiative to prevent methicillin-resistant Staphylococcus aureus infections. N Engl J Med 2011; 364: 1419-1430

3 Gurieva T, Bootsma MC, Bonten MJ. Successful Veterans Affairs initiative to prevent methicillin-resistant Staphylococcus aureus infections revisited. Clin Infect Dis 2012; 54: $1618-1620$

4 Huang SS, Septimus E, Kleinman K et al. Targeted versus universal decolonization to prevent ICU infection. N Engl J Med 2013: DOI: 10.1056/NEJMoa1207290

5 Edmond MB, Wenzel RP. Screening inpatients for MRSA (case closed. N Engl J Med 2013: DOI: 10.1056/NEJMe1304831

6 Wenzel RP, Edmond MB. Infection control: the case for horizontal rather than vertical interventional programs. Int J Infect Dis 2010; 14 : 043 - S5

7 Schulze-Röbbecke R. Standardmaßnahmen zur Prävention der Übertragung nosokomialer Infektionen - Standardhygiene, Basishygiene. Krankenhaushygiene up2date 2009; 4: 193 205

8 Schulze-Röbbecke R. Bündel zur Prävention nosokomialer Infektionen. Krankenhaushygiene up2date 2011; 6: 9-23

9 Morgan DJ, Diekema DJ, Sepkowitz K et al. Adverse outcomes associated with contact precautions: a review of the literature. Am J Infect Control 2009; 37: 85-93

10 Kappstein I, van der Mühlen K, Meschzan D et al. Prävention von MRSA-Übertragungen: Standardhygiene statt Isolierung. Chirurg 2009; 80: 49-61 